\title{
Orbital Parallaxes of Wide Orbit Binary Pulsars and their implications
}

\author{
Sergei M. Kopeikin
}

Laboratory of Astronomy and Geophysics, Hitotsubashi University, Kunitachi, Tokyo 186, Japan

and

Astro Space Centre, RAS FIAN, Leninskii Prospect, 53, Moscow 117924, Russia

A complete theoretical description of the parallactic terms in the timing formula for a wide orbit binary pulsar is presented. It is shown that the terms depend not only on the annual motion of Earth but also on the orbital motion of the binary pulsar as well. It is proved that measurements of the effect of orbital parallax open a new way in determination of pulsar distances, and can be used for measurement of companion masses as well. Estimates of expected magnitudes of the corresponding timing signals for the most appropriate binary pulsar candidates are tabulated, and prospects for the measurement of orbital parallaxes are discussed.

\section{References}

S.M. Kopeikin, 1995, ApJ, Vol.439, L5 